\title{
Two novel mutations in the ALPL gene of unrelated Chinese children with Hypophosphatasia: case reports and literature review
}

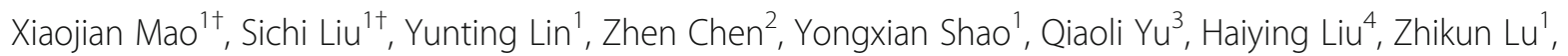
Huiyin Sheng ${ }^{1}$, Xinshuo Lu', Yonglan Huang ${ }^{1}$, Li Liü ${ }^{1 *}$ and Chunhua Zeng ${ }^{1 *}$ (I)

\begin{abstract}
Objective: Hypophosphatasia (HPP) is an inherited disorder of defective skeletal mineralization caused by mutations in the ALPL gene that encodes the Tissue Non-specific Alkaline Phosphatase (TNSALP). It is subdivided into six forms depending on the age of onset: perinatal lethal, prenatal benign, infantile, childhood, adult, and odonto HPP. Among these, infantile HPP is characterized by early onset and high frequency of lethal outcome. Few studies have reported the phenotype and genetic characteristics of HPP in Chinese children.

Case presentation: Three forms of HPP were identified in four unrelated patients from four different Chinese families, including one lethal infantile (patient 1), two childhood (patient 2 and 3) and one odonto HPP (patient 4). Six variants in the ALPL gene were identified, including five missense mutations and one frameshift mutation. Of which, none were reported previously in the Chinese population, and two were novel (c.359G > C: p.G120A and c.1017dupG: p.H340AfsX3). Patient 1 carrying a novel homozygous (c.359G > C) mutation showed respiratory distress and pneumonia at first day of his life. He presented nearly negligible level of serum ALP activity, overall skeletal hypominaralization and died at 3 months old. Patient 2, 3 and 4 were compound heterozygotes with decreased serum ALP activity. Patient 2 and 3 presented premature loss of deciduous teeth, muscle weakness and bone pain, whereas patient 4 had early loss of deciduous teeth only. All four pedigrees exhibited autosomal recessive pattern of inheritance.

Conclusions: In this study, six mutations in the ALPL gene were found in four Chinese HPP patients, two of which were novel: c.359G > C in exon 5 and c.1017dupG in exon 10. Our results strongly indicated that the novel mutation c.359G > C might be disease-causing and associated with severe infantile form of HPP.
\end{abstract}

Keywords: Hypophosphatasia, Alkaline phosphatase, Mutation, Hypomineralization

\section{Background}

Hypophosphatasia (HPP) is a rare hereditary disorder caused by loss of function mutations in the ALPL gene that encodes the Tissue Nonspecific Alkaline Phosphatase (TNSALP), one of the alkaline phosphatase (ALP) family members [1-3]. TNSALP is predominantly expressed in the liver, skeleton, kidney and teeth [4]. Its specific

\footnotetext{
*Correspondence: liliuchina@qq.com; chunhuazeng@hotmail.com

${ }^{+}$Xiaojian Mao and Sichi Liu contributed equally to this work.

1 Department of Genetics and Endocrinology, Guangzhou Women and

Children's Medical Center, Guangzhou Medical University, 9 Jinsui Rd,

Guangzhou 510623, China

Full list of author information is available at the end of the article
}

function is to cleave the extracellular substrates including inorganic pyrophosphate (PPi), pyridoxal-5-phosphate (PLP) and phosphoethanolamine (PEA) [5]. The deficiency of TNSALP causes the extracellular accumulation of $\mathrm{PPi}$, a potent inhibitor of mineralization, resulting in the defective teeth and bones.

HPP is clinically characterized by decreased level of serum ALP activity and defective skeletal mineralization. It is subdivided into six forms depending on the age at diagnosis: perinatal lethal, prenatal benign, infantile, childhood, adult, and odonto HPP [6]. The patients with lethal perinatal form show markedly impaired mineralization in utero,

(c) The Author(s). 2019 Open Access This article is distributed under the terms of the Creative Commons Attribution 4.0 International License (http://creativecommons.org/licenses/by/4.0/), which permits unrestricted use, distribution, and reproduction in any medium, provided you give appropriate credit to the original author(s) and the source, provide a link to the Creative Commons license, and indicate if changes were made. The Creative Commons Public Domain Dedication waiver (http://creativecommons.org/publicdomain/zero/1.0/) applies to the data made available in this article, unless otherwise stated. 
whereas the patients with infantile form mostly present respiratory complications, widespread demineralization and rachitic symptoms during the first 6 months of life, both of which are defined as severe forms of HPP [6]. The childhood form of HPP displays milder symptoms between 6 months and 18 years with premature loss of primary teeth, delayed walking, short stature and bone deformities. As one of the mildest forms of HPP, odonto HPP is characterized by premature exfoliation of primary and/or severe dental caries without abnormalities of the skeletal system.

Both autosomal dominant and recessive transmission have been shown in HPP. In general, the more severe the HPP is, the more often it could be recessive inheritance. For instance, lethal perinatal form and most infantile forms of HPP are recessively inherited, whereas less severe forms, including perinatal benign, childhood, odonto and adult forms of HPP, show both dominant and recessive inheritance [7]. It has been reported that severe HPP has lower prevalence $(1 / 300,000)$ than that of less severe forms of HPP (1/6370) [8]. Further, more severe forms of the HPP present lower serum AP activity levels. The studies all indicated that the severity of the HPP is correlated with the activity level of ALP, which is encoded by the ALPL gene [9].

The diagnosis of HPP is based on low level of serum ALP activity and genetic testing of the ALPL gene mutations. To date, a total of 390 disease-causing mutations in the ALPL gene have been reported, most of which are missense mutations (70.3\%) (http://www.sesep.uvsq.fr/ 03_hypo_mutations.php). It has been suggested that large variety of mutations results in variable deficiency of ALP activity and distinct clinical phenotypes $[6,10]$. Although genotype-phenotype correlation has been observed, it needs more clinical and genetic data to support. Particularly, there are few pediatric HPP patients reported in the Chinese population.

In the present study, we characterized the distinct clinical and mutational features of four unrelated Chinese children with different forms of HPP, and explored the correlations between the phenotype and genotype in a lethal infantile HPP with a novel homozygous ALPL mutation.

\section{Case presentation \\ Clinical features}

The clinical phenotypes of all four patients with HPP (three males and one female) from four unrelated families are summarized in Table 1 and Fig. 1. They were all born to nonconsanguineous parents. They were initially referred to our clinic due to variable clinical manifestations, including growth failure, premature loss of teeth and rachitic symptoms. With biochemical tests, all patients showed remarkably decreased levels of ALP activity (Table 1). All patients were then suspected and finally diagnosed as HPP with confirmation of diseasecausing mutations in the ALPL gene.

Patient 1 (PA-1) was delivered at term by c-section with a birth weight of $3000 \mathrm{~g}$. He experienced respiratory distress and newborn pneumonia soon after he was born. He presented with feeding difficulty, seizure, poor head control and failure to thrive during the first 2 months of his life. He showed underweight, short stature, enlargement of the anterior fontanelle, and developmental delay when he was diagnosed at 2 months old. His serum ALP activity was almost undetectable (Table 1). X-ray showed general hypomineralization, deformity of long bones, flared metaphyses and hypolucent mid-metaphyses (Fig. 1a and b). He died of pneumonia and respiratory failure at 3 months old. Patient 1 was classified as infantile HPP (Table 1).

Patient 2 (PA-2) began to present premature loss of the deciduous teeth and muscle weakness at 1 year old. She had waddling gait and delay of walking. She had lost most of her deciduous teeth and only reserved seven teeth when she came to the clinic at 2 years old (Fig. 1c and d). Very low level of serum ALP activity (42 U/L) suggested the diagnosis of HPP (Table 1). Panoramic radiographs showed premature loss of most of deciduous teeth, taurodontism in the deciduous molars and maldevelopment of permanent teeth (Fig. 1e). X-ray of wrist and knee revealed flared metaphyses and bone destruction in distal femur and proximal tibia (Fig. If and g). Patient 2 was diagnosed as childhood HPP at 2 years and 5 months old (Table 1 ).

Patient 3 (PA-3) began to present joint swelling, bone pain and muscle weakness at 1 year old. He experienced delay of walking and early loss of deciduous teeth. His symptoms of joint swelling and bone pain were not relieved at 8 years old. He had body weight and height within normal ranges. Serum ALP activity was $41 \mathrm{U} / \mathrm{L}$ at 4 years old and $67 \mathrm{U} / \mathrm{L}$ at 8 years old. Panoramic X-ray showed taurodontism, reduced alveolar bone, and enlarged pulp chambers and root canals (Fig. 1j). The Xray revealed hypomineralization at 4 years old (Fig. 1h, i) and bone destruction in the proximal humerus at 8 years old (Fig. 1k, l). Patient 3 was diagnosed as childhood HPP at 8 years old (Table 1 ).

Patient 4 (PA-4) experienced premature loss of deciduous teeth since he was one and half years old. The physical examination was unremarkable, other than the absence of the upper and lower anterior incisor and canine teeth (Fig. 1m). Serum ALP activity was $42 \mathrm{U} / \mathrm{L}$ at diagnosis when he was 4 years old. Panoramic X-ray showed a reduced alveolar bone of whole dentition (Fig. 1n). His wrist and knee X-ray revealed normal growth plates without any evidence of defective bones (Fig. 10 and p). Patient 4 was diagnosed as odonto HPP at 4 years old (Table 1 ). 
Table 1 Clinical features of 4 HPP patients at diagnosis

\begin{tabular}{|c|c|c|c|c|}
\hline Patient & PA-1 & PA-2 & PA-3 & PA-4 \\
\hline Gender & M & F & M & M \\
\hline Age of onset & $1 d$ & $1 \mathrm{y}$ & $1 y$ & $1.5 y$ \\
\hline Age at diagnosis & $2 m$ & $2.4 y$ & $8 y$ & $4 y$ \\
\hline Form of HPP & Infantile & Childhood & Childhood & Odonto \\
\hline Serum ALP (U/I) (reference 118-390) & 5 & 42 & 67 & 42 \\
\hline Calcium (mmol/L) (reference 2.2-2.7) & 3.1 & 2.4 & 2.5 & 2.2 \\
\hline Serum Phosphate (mmol/L) (reference 1.3-1.9) & 2.1 & 2 & 1.7 & 1.66 \\
\hline Serum PTH (pmol/L) (reference 1.2-7.1) & 0.32 & 0.32 & 1.56 & 0.36 \\
\hline 25-hydroxyvitamin $\mathrm{D}(\mathrm{nmol} / \mathrm{L})$ (reference $50-150$ ) & na & 78.2 & 54.4 & 71 \\
\hline Height (SD) & $<-2 S D$ & OSD & $0 \sim-1 S D$ & $0 \sim-1 S D$ \\
\hline Failure to thrive & Y & N & N & $\mathrm{N}$ \\
\hline Waddling gait & NA & Y & Y & $\mathrm{N}$ \\
\hline Joint pain & $\mathrm{N}$ & N & Y & $\mathrm{N}$ \\
\hline Early loss of deciduous teeth & NA & Y & Y & Y \\
\hline Hypomineralisation & Y & Y & Y & Y \\
\hline Loss of bone & Y & Y & Y & N \\
\hline Deformity of long bones & Y & N & $\mathrm{N}$ & $\mathrm{N}$ \\
\hline Flared metaphyses & Y & Y & Y & $\mathrm{N}$ \\
\hline Nephrocalcinosis & $\mathrm{N}$ & N & $\mathrm{N}$ & $\mathrm{N}$ \\
\hline Enzyme replacement & $\mathrm{N}$ & N & $\mathrm{N}$ & $\mathrm{N}$ \\
\hline Outcome & Dead & Alive & Alive & Alive \\
\hline
\end{tabular}

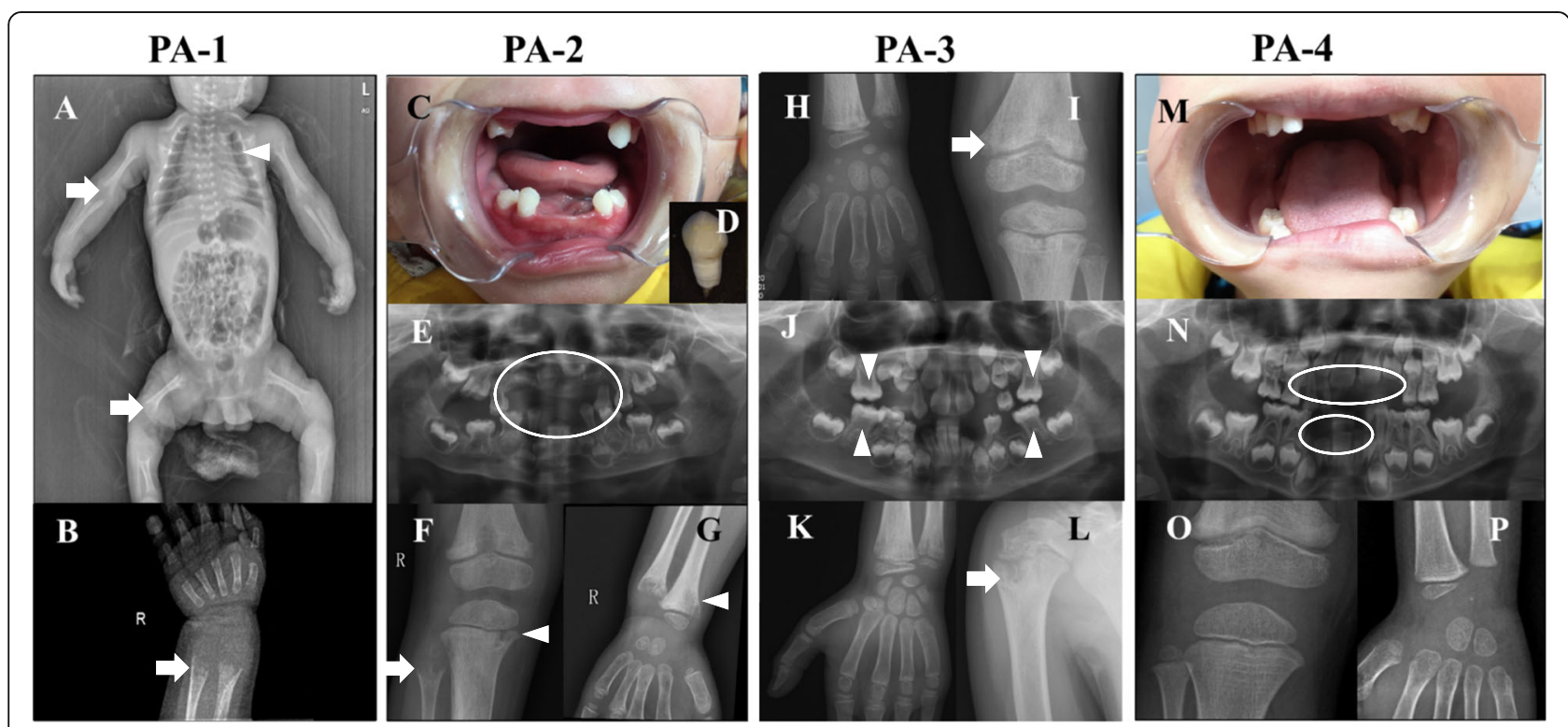

Fig. 1 Photos and X-rays of teeth and bones in 4 HPP children with HPP. a \& b X-rays show general hypomineralization, gracile ribs (arrowhead), small clavicles, shortened long bones and flared metaphysis (arrow) in patient 1 (PA-1) at 2-month-old; $\mathbf{c}$, d \& e Premature loss of deciduous tooth (circle) in Patient 2 (PA-2) at 2-year-old; $\mathbf{f} \& \mathbf{g}$ X-rays show flared metaphysis (arrow), and bone destruction (arrowhead) in PA-2; h \& i X-ray shows hypomineralization (arrow) in PA-3 at 4-year-old; $\mathbf{j}$ Panoramic X-ray in PA-3 at 8-year-old shows decreased mineralization of the alveolar bone, enlarged pulp chambers (arrowhead) and alveolar bone resorption; $\mathbf{k} \& \mathbf{I}$ Bone destruction in the proximal humerus (arrow) of PA-3 at 8year-old; $\mathbf{m} \& \mathbf{n}$ Premature loss of deciduous tooth (circle) in Patient 4 (PA-4) at 4-year-old; $\mathbf{o} \& \mathbf{p}$ X-ray didn't show significant bone abnormality 


\section{Mutational analysis}

In order to confirm the diagnosis of HPP and correlate the phenotype with specific genotype, mutational analyses of the ALPL gene were performed in all four patients and their parents except the mother of patient 1 was not involved in this study. The ALPL variants in patient 1 and patient 3 were identified using next generation sequencing and confirmed with Sanger sequencing, whereas the variants in patient 2 and patient 4 were identified and confirmed by direct Sanger sequencing. A 3D structural modeling of the TNSALP constructed based on its sequence homology to the placental isozyme (PDB ID: 1EW2), was used to locate the missense mutation [11].

Six different variants were identified in the ALPL gene in our four patients, including five missense variants and one small insertion variant (Table 2). All variants were inherited from their unaffected parents except patient 1 (Fig. 2). As the most severe and lethal infantile HPP in the present study, patient 1 carried a novel homozygous variant of c.359G > C (p.G120A) at exon 5 of the ALPL gene, which was predicted to be disease-causing by insilico analysis of bioinformatics software. His father demonstrated a heterozygous status, while his mother was not involved in this study. To further investigate the effect of the novel missense mutation of c.359G > C, a 3D structure modeling of TNSALP was used to locate the residue, indicating that the residue p.G120A was located in the secondary structure of the TNSALP homodimer interface.

Patient 1 (PA-1) was homozygote, whose father (PA$1 \mathrm{~F})$ was heterozygous carrier, but his mother's genotype was unknown (she was not detected). All of other 3 patients (PA-2, PA-3 and PA-4) were compound heterozygote whose variants were inherited from their parents. Arrows indicated the mutated sites. F means father and $M$ means mother.

\section{Literature review}

In order to provide a comprehensive overview of Chinese patients with HPP, we reviewed all publications regarding Chinese HPP cases in the PubMed database (https://www. ncbi.nlm.nih.gov/pubmed) and the $A L P L$ gene mutations database (http://www.sesep.uvsq.fr/03_hypo_mutations. php) [12-20].

The full spectrum of $A L P L$ gene mutations in reported Chinese HPP patients is presented in Fig. 3. A total of 26 mutations from 15 Chinese families were previously reported, including 20 missense mutations, 4 small deletion mutations, one small insertion and one splice site mutation. The 26 mutations were distributed throughout all 12 exons of the ALPL gene except for exon 1 and 8, with mutations most often located at exon $5(27.6 \%, 8 /$ 29). Three mutations (c. $407 \mathrm{G}>\mathrm{A}$, c. $1162 \mathrm{~T}>\mathrm{C}$ and c. $1166 \mathrm{C}>\mathrm{A}$ ) were detected twice. No hot mutation was identified. The most common form of HPP in Chinese patients was childhood HPP, followed by adult HPP, whereas only one patient with onset of age at 1 month old was reported as infantile HPP. No lethal HPP has been reported in Chinese patients.

In the present study, three clinical forms of HPP were diagnosed in four Chinese children, which were infantile, childhood and odonto HPP. All six mutations (c.203C > T, c. $212 \mathrm{G}>\mathrm{A}$, c. $359 \mathrm{G}>\mathrm{C}$, c. $571 \mathrm{G}>\mathrm{A}$, c. $979 \mathrm{~T}>\mathrm{C}$ and c.1017dupG) were first reported in the Chinese population. Of which, c.359G $>\mathrm{C}$ and c.1017dupG were novel mutations. Patient 1 carrying homozygous c.359G >C exhibited severe phenotype of lethal infantile HPP.

\section{Discussion and conclusions}

The ALPL gene encoding TNSALP was located on chromosome 1p34-36. HPP is caused by loss of function mutations in the ALPL gene. The first case of HPP was reported in 1948 and the $A L P L$ gene mutation was first reported in 1988 [1, 21]. HPP presents a heterogeneous phenotype ranging from life threatening to asymptomatic presentation. In general, the pattern of inheritance in HPP is autosomal recessive. However, HPP may also be transmitted by autosomal dominant and incomplete penetrance of dominant transmission [22]. The severe forms of HPP usually transmit by autosomal recessive, while milder forms of HPP more often present with autosomal dominant with variable expressivity. These heterogeneous clinical features and inheritance patterns

Table 2 ALPL gene mutations in 4 HPP Chinese patients

\begin{tabular}{|c|c|c|c|c|c|c|}
\hline Patient & Genotype & Exon & Nucleotide change & Amino acid change & Mutation type & Reported previously \\
\hline PA-1 & homozygous & 5 & c.359G $>C$ & p.G120A & Missense & Novel \\
\hline \multirow[t]{2}{*}{ PA-2 } & Compound heterozygous & 4 & $c .212 \mathrm{G}>\mathrm{A}$ & p.R71H & Missense & Reported \\
\hline & & 6 & c. $571 \mathrm{G}>\mathrm{A}$ & p.E191K & Missense & Reported \\
\hline \multirow[t]{2}{*}{ PA-3 } & Compound heterozygous & 4 & $c .203 C>T$ & p.T68M & Missense & Reported \\
\hline & & 6 & c. $571 \mathrm{G}>\mathrm{A}$ & p.E191K & Missense & Reported \\
\hline \multirow[t]{2}{*}{ PA-4 } & Compound heterozygous & 9 & c.979T>C & p.F327L & Missense & Reported \\
\hline & & 10 & c.1017dupG & p.H340AfsX3 & Frameshift & Novel \\
\hline
\end{tabular}




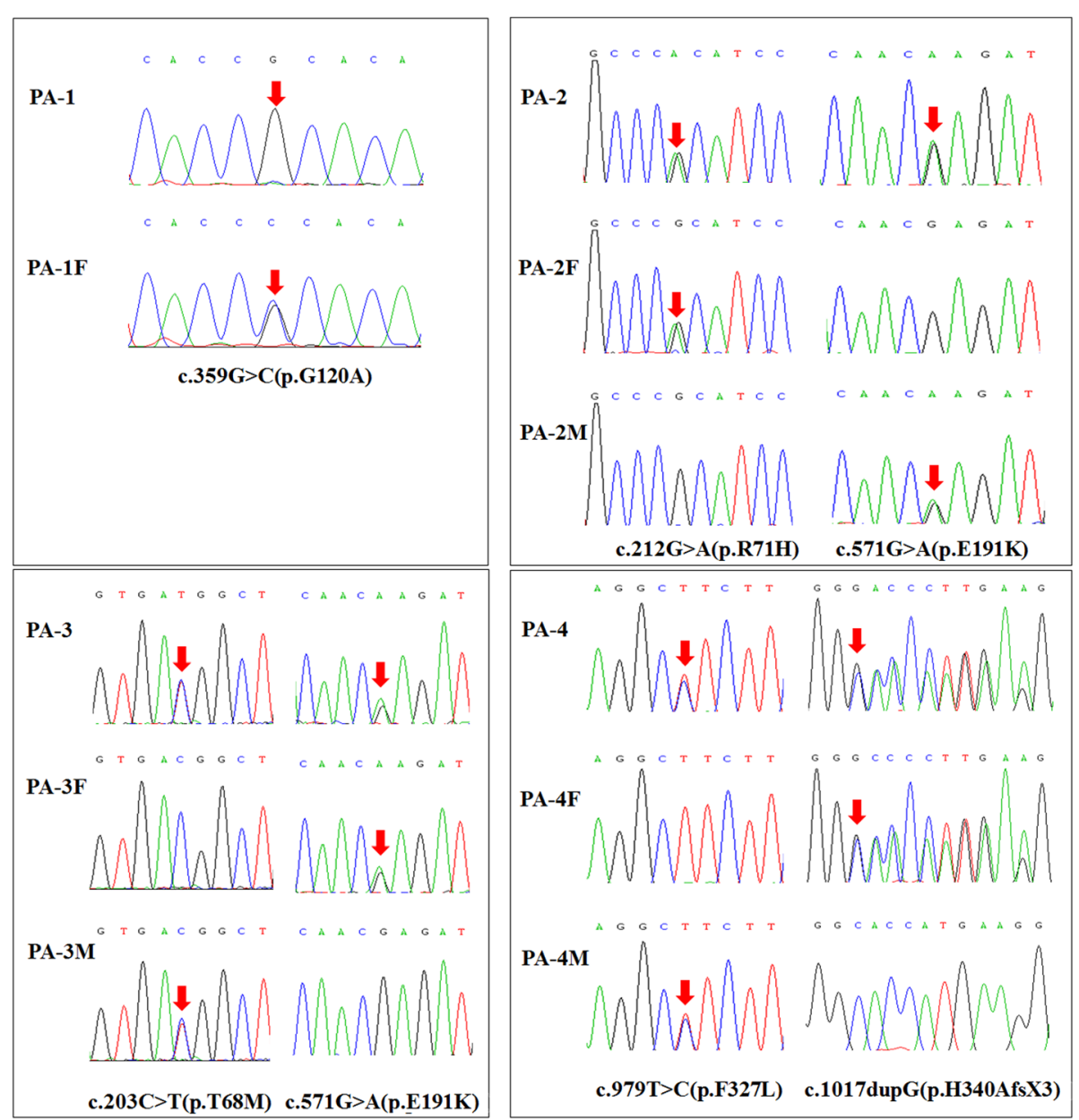

Fig. 2 Chromatograms of 6 ALPL mutations identified in the current study. Each frame represents the sequencing results of each pedigree

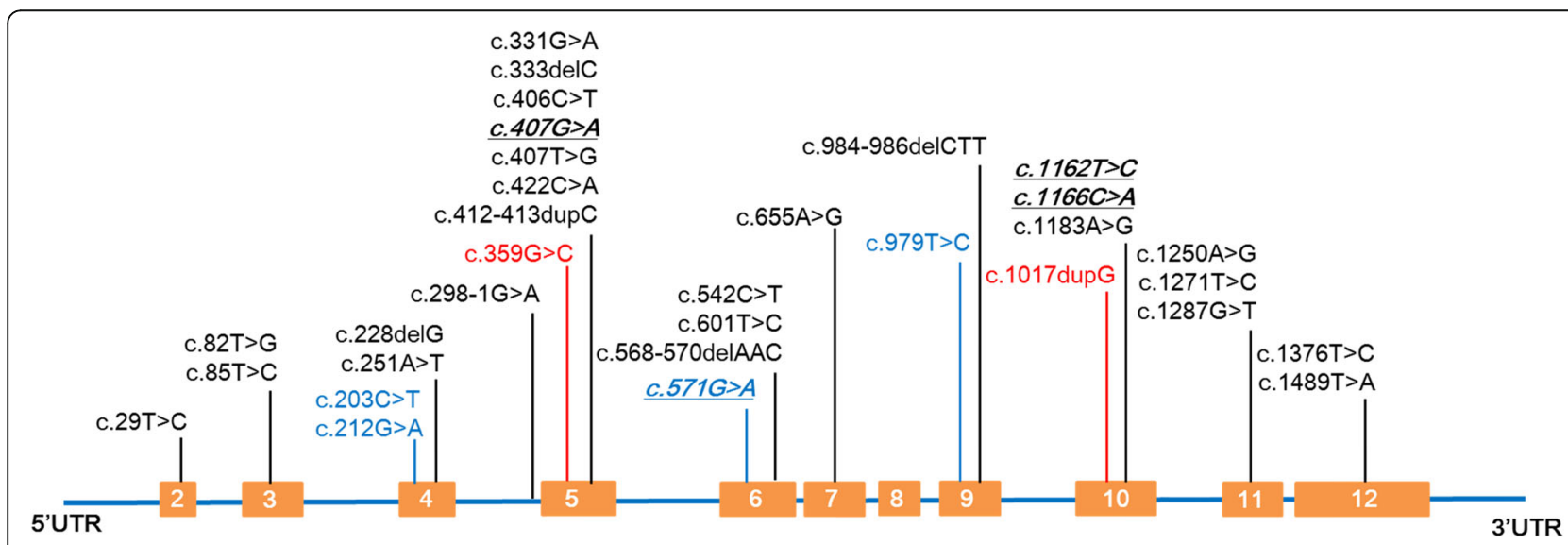

Fig. 3 Schematic drawing of the ALPL gene mutations identified in Chinese HPP patients. The position of all identified mutations are indicated. The mutations reported previously are indicated in black, while six mutations identified in the present study are indicated in red and blue. The red color highlights the novel mutations, and the blue color represents the pathogenic mutations reported previously, whereas italic font with underline represents the mutations detected in two patients 
result in the difficulty of timely diagnosis and challenging of genetic counselling [23, 24].

In the present study we investigated four Chinese children affected by three forms of HPP, which were lethal infantile, childhood and odonto HPP. All patients follow autosomal recessive inheritance pattern. They presented clinical symptoms early at 1 day old (patient 1 ) or at 1 year old (patient 2, 3 and 4). All four patients had low serum ALP activity. As reported, patients with perinatal or infantile HPP have high morbidity and mortality in the first 5 years of life [7]. Patient 1 was a typical infantile form of HPP who presented respiratory distress, nearly undetectable ALP activity and general skeletal hypomineralization. He died of respiratory failure at 3 months old, therefore was diagnosed as lethal infantile form of HPP. To the best we know, this was the first case of lethal infantile HPP reported in the Chinese population. Recent researches revealed that a rapidly worsening clinical course often occurs in prenatal [25] or infantile HPP mainly due to respiratory compromise [7]. However, further investigation is need to clarify the mechanism.

It has been reported that diagnostic delay is common due to limited awareness of HPP [26]. Patient 2 and 3 were Childhood HPP who presented premature loss of deciduous teeth and muscle weakness at 1 year old. Patient 3 also had joint swelling and bone pain starting at 1 year old, however, he was confirmed with HPP at 8 years old. Patient 4 , although his early loss of deciduous teeth started at 1 year old, was diagnosed at 4 years old. These observation demonstrated that some HPP cases may not be recognized well and managed timely.

Molecular diagnosis provides great advantage to confirm the diagnosis of HPP. HPP is caused by a loss of function mutation in the ALPL gene encoding TNSALP. It has been reported that few mutations may be frequent in particular populations [23]. For instance, c.1559delT and p.F327 L are two common mutations in the Japanese population, whereas c.571G > A (p.E191 K) is identified in half of European patients with moderate HPP [27, 28]. To elucidate the mutational characteristics of the $A L P L$ gene in the Chinese population, we reviewed all reported Chinese HPP cases in the literature. We found that the most mutations in Chinese HPP patients were missense variants located in exon 5 in the ALPL gene. No frequent mutations were recognized (Fig. 3). In the present study, six $A L P L$ mutations, including five missense mutations and one splicing mutation, were identified. All of these mutations were first reported in the Chinese population.

It is considered that the variety of $A L P L$ mutations results in highly variable clinical expressivity, resulting in difficulty to assess the severity of a novel mutation in the ALPL gene [15]. The severity of HPP symptoms was correlated with the level of ALP activity affected by the ALPL mutation [29]. Severe HPP often exhibit very low level of ALP activity, whereas mild HPP usually retain most ALP activity. Severe form manifests early, whereas mild form may be diagnosed in adulthood [30]. Patient 1 was a homozygote of c.359G > C which has not been previously described in the literature. However, typical clinical features, very low level of serum ALP activity, overall hypomineralization and homozygote status all were consistent with severe infantile form of HPP, indicating the variant c.359G $>\mathrm{C}$ seems to be at a crucial position of ALP protein. Further investigation revealed that the variant c.359G $>\mathrm{C}$ (p.G120A) is located in the homodimer interface, a crucial site of secondary structure in the TNSALP and highly conserved throughout many species. Thus, the novel mutation c.359G >C was strongly indicated to be disease-causing and related to severe infantile form of HPP. Further functional study of the mutation c.359G $>\mathrm{C}$ is needed.

Patient 2, 3 and 4 were compound heterozygote in the ALPL gene. Patient 2, the childhood HPP, had been identified with two known pathogenic variants, c.212G > A (p.R71H) at exon 4 and c.571G > A (p.E191K) at exon 6 [31, 32]. As another childhood HPP, Patient 3 carried the identical c.571G > A (p.E191K) variant of Patient 2 and another pathogenic c.203C $>$ T (p.T68 M) variant at exon 4 which was also reported previously [33]. Interestingly, the variant c.571G > A (p.E191 K) in Patient 2 and 3 , was the common mutation reported in European population with moderate HPP. Patient 4 was the mildest odonto HPP in the present study. He demonstrated c.979 T > C (p.G120A) and c.1017dupG (p. H340AfsX3). The c.979 T > C (p.G120A) variant was pathogenic reported previously [34], whereas the novel variant c.1017dupG (p.H340AfsX3) was predicted to result in a translation frameshift and premature protein termination (p. H340AfsX3).

Some limitations exist in the present study. First, the number of HPP patients is not big enough to reveal the phenotype-genotype correlations. Second, PLP, the best markers of HPP, was not detected in the present study since the method is still not available in our center. Future study will be focused on collecting more data to reveal the correlation between phenotype and genotype of HPP in the Chinese population.

Currently enzyme replacement therapy with asfotase alfa (Strensiq ${ }^{\text {ma }}$, USA) is currently the only approved treatment for HPP. Asfotase alfa can restore normal ALP levels and prevented skeletal and dental manifestations of HPP. Unfortunately it has been approved in many countries but not in China. None of the patients in the present study have received asfotase alfa for treatment. 
In the present study, we described the clinical and genetic characteristics of HPP in four unrelated Chinese pedigrees who were affected with infantile, childhood and odonto forms of HPP. All patients followed autosomal recessive inheritance pattern. Six mutations in the ALPL gene were identified including four known mutations and two novel mutations. The novel missense mutation (c.359G > C) caused the decrease of ALP activity and was related to lethal infantile form of HPP. The study expanded knowledge about the characteristics of HPP.

\section{Abbreviations}

ALP: Alkaline phosphatase; HPP: Hypophosphatasia;

PEA: Phosphoethanolamine; PLP: Pyridoxal-5-phosphate; PPi: Inorganic pyrophosphate; TNSALP: Tissue Non-specific Alkaline Phosphatase

\section{Acknowledgements}

The authors thank all the patients and their family members for their involvement in the present study. The authors thank Dr. Guoqing Hou at University of Michigan for his kind suggestions.

\section{Authors' contributions}

This work was carried out in collaboration between all authors. CZ, LL, YH and XM designed the study; SC acquired the data; YL, YS, HL, HS, ZL performed experiments. $X M, X L$ and $C Z$ analyzed the data; $C Z, X M, S L, Z C$, and QY wrote the manuscript; $L L$ and $Y H$ gave technical support and conceptual advice. All authors read and approved the final manuscript.

\section{Funding}

This work was supported by Medical Scientific Research Foundation of Guangdong Province, China (A2018546), the Research Grants from Guangzhou Women and Children's Medical Center (5001-4001003), and the Youth Pilot Project from Guangzhou Institute of Pediatrics (YIP-2016-010). Funders were not involved in data analysis or interpretation.

\section{Availability of data and materials}

Not applicable. We are submitting the data together with this manuscript.

\section{Ethics approval and consent to participate}

All authors hereby declare that all experiments have been examined and approved by the Medical Ethics Committee for Clinical Ethical Review, Guangzhou Women and Children's Medical Center (No.37701) and have therefore been performed in accordance with the ethical standards laid down in the 1964 Declaration of Helsinki. A written informed consent was obtained from the parents before inclusion into the study.

\section{Consent for publication}

The parent of each patient provided written informed consent for publication of medical data and genetic data.

\section{Competing interests}

The authors declare that they have no competing interests.

\section{Author details}

${ }^{1}$ Department of Genetics and Endocrinology, Guangzhou Women and Children's Medical Center, Guangzhou Medical University, 9 Jinsui Rd, Guangzhou 510623, China. ${ }^{2}$ Department of Radiology, Guangzhou Women and Children's Medical Center, Guangzhou Medical University, 9 Jinsui Rd., Guangzhou 510623, China. ${ }^{3}$ Department of Dentistry, Guangzhou Women and Children's Medical Center, Guangzhou Medical University, 9 Jinsui Rd., Guangzhou 510623, China. ${ }^{4}$ Clinical Laboratory, Guangzhou Women and Children's Medical Center, Guangzhou Women and Children's Medical Center, Guangzhou Medical University, 9 Jinsui Rd., Guangzhou 510623, China.
Received: 2 July 2019 Accepted: 24 October 2019

Published online: 25 November 2019

\section{References}

1. Rathbun JC. Hypophosphatasia; a new developmental anomaly. Am J Dis Child. 1948;75:822-31.

2. Rathbun JC, Macdonald JW, Robinson HM, Wanklin JM. Hypophosphatasia: a genetic study. Arch Dis Child. 1961;36:540-2.

3. Whyte MP, Coburn SP, Ryan LM, Ericson KL, Zhang F. Hypophosphatasia: biochemical hallmarks validate the expanded pediatric clinical nosology. Bone. 2018:110:96-106.

4. Whyte MP. Hypophosphatasia: an overview for 2017. Bone. 2017;102:15-25.

5. Macfarlane JD, Poorthuis BJ, Mulivor RA, Caswell AM. Raised urinary excretion of inorganic pyrophosphate in asymptomatic members of a hypophosphatasia kindred. Clin Chim Acta. 1991;202:141-8.

6. Mornet E. Hypophosphatasia. Orphanet J Rare Dis. 2007;2:40.

7. Whyte MP, Leung E, Wilcox WR, Liese J, Argente J, Martos-Moreno GA, et al. Natural History of Perinatal and Infantile Hypophosphatasia: A Retrospective Study. J Pediatr. 2019:209:116-124.e4.

8. Mornet E. Genetics of hypophosphatasia. Arch Pediatr. 2017;24:5s51-6.

9. Mornet E. Hypophosphatasia Metabolism. 2018;82:142-55.

10. Zurutuza L, Muller F, Gibrat JF, Taillandier A, Simon-Bouy B, Serre JL, et al. Correlations of genotype and phenotype in hypophosphatasia. Hum Mol Genet. 1999:8:1039-46.

11. Le D, Le Du MH, Millan JL. Structural evidence of functional divergence in human alkaline phosphatases. J Biol Chem. 2002;277:49808-14.

12. Xu L, Pang $Q$, Jiang $Y$, Wang $O$, Li M, Xing X, et al. Four novel mutations in the ALPL gene in Chinese patients with odonto, childhood, and adult hypophosphatasia. Biosci Rep. 2018;38.

13. Chen $B$, Li L, Ren $W$, Yi L, Wang Y, Yan F. A novel missense mutation in the ALPL gene causes dysfunction of the protein. Mol Med Rep. 2017;16:710-8.

14. Yang H, Wang L, Geng J, Yu T, Yao RE, Shen Y, et al. Characterization of six missense mutations in the tissue-nonspecific alkaline phosphatase (TNSALP) gene in Chinese children with hypophosphatasia. Cell Physiol Biochem. 2013:32:635-44

15. Wei KW, Xuan K, Liu YL, Fang J, Ji K, Wang X, et al. Clinical, pathological and genetic evaluations of Chinese patients with autosomal-dominant hypophosphatasia. Arch Oral Biol. 2010;55:1017-23.

16. Li DF, Lan D, Zhong JZ, Dewan RK, Xie YS, Yang Y. Infantile hypophosphatasia caused by a novel compound heterozygous mutation: a case report and pedigree analysis. Zhongguo Dang Dai Er Ke Za Zhi. 2017; 19:539-44.

17. Bai Y, Liu N, Yang J, Guo Y, Kong XD. Mutation analysis for two hypophosphatasia families with targeted next-generation sequencing. Zhonghua Yi Xue Za Zhi. 2016;96:3718-23.

18. Zhao Z, Xia WB, Xing XP, Li M, Wang O, Jiang $Y$, et al. Infantile hypophosphatasia due to mutations in the tissue-nonspecific alkaline phosphatase gene. Zhonghua Nei Ke Za Zhi. 2013;52:824-8.

19. Zhang H, Ke YH, Wang C, Yue H, Hu WW, Gu JM, et al. Identification of the mutations in the tissue-nonspecific alkaline phosphatase gene in two Chinese families with hypophosphatasia. Arch Med Res. 2012;43:21-30.

20. Zhou W, Li LL, Chen B, Yan FH, Li HX. Hypophosphatasia: a case report and gene mutation analysis. Zhonghua Kou Qiang Yi Xue Za Zhi. 2017;52:578-9.

21. Weiss MJ, Cole DE, Ray K, Whyte MP, Lafferty MA, Mulivor RA, et al. A missense mutation in the human liver/bone/kidney alkaline phosphatase gene causing a lethal form of hypophosphatasia. Proc Natl Acad Sci U S A. 1988;85:7666-9.

22. Hu JC, Plaetke R, Mornet E, Zhang C, Sun X, Thomas HF, et al. Characterization of a family with dominant hypophosphatasia. Eur J Oral Sci. 2000:108:189-94.

23. Mornet E. Molecular genetics of Hypophosphatasia and phenotypegenotype correlations. Subcell Biochem. 2015;76:25-43.

24. Ozono K, Michigami T. Hypophosphatasia now draws more attention of both clinicians and researchers: a commentary on Prevalence of c. 1559delT in ALPL, a common mutation resulting in the perinatal (lethal) form of hypophosphatasias in Japanese and effects of the mutation on heterozygous carriers. J Hum Genet. 2011;56:174-6.

25. Taketani T, Onigata K, Kobayashi H, Mushimoto Y, Fukuda S, Yamaguchi S. Clinical and genetic aspects of hypophosphatasia in Japanese patients. Arch Dis Child. 2014;99:211-5. 
26. Hogler W, Langman C, da Silva HG, Fang S, Linglart A, Ozono K, et al. Diagnostic delay is common among patients with hypophosphatasia: initial findings from a longitudinal, prospective, global registry. BMC Musculoskelet Disord. 2019:20:80

27. Michigami T, Uchihashi T, Suzuki A, Tachikawa K, Nakajima S, Ozono K. Common mutations F310L and T1559del in the tissue-nonspecific alkaline phosphatase gene are related to distinct phenotypes in Japanese patients with hypophosphatasia. Eur J Pediatr. 2005;164:277-82.

28. Herasse M, Spentchian M, Taillandier A, Mornet E. Evidence of a founder effect for the tissue-nonspecific alkaline phosphatase (TNSALP) gene E174K mutation in hypophosphatasia patients. Eur J Hum Genet. 2002;10:666-8.

29. Linglart A, Salles JP. Hypophosphatasia: the contribution of imaging. Arch Pediatr. 2017;24:5s74-9.

30. Tenorio J, Alvarez I, Riancho-Zarrabeitia L, Martos-Moreno GA, Mandrile G, de la Flor Crespo M, et al. Molecular and clinical analysis of ALPL in a cohort of patients with suspicion of Hypophosphatasia. Am J Med Genet A. 2017; 173:601-10.

31. Taillandier A, Lia-Baldini AS, Mouchard M, Robin B, Muller F, Simon-Bouy B, et al. Twelve novel mutations in the tissue-nonspecific alkaline phosphatase gene (ALPL) in patients with various forms of hypophosphatasia. Hum Mutat. 2001;18:83-4.

32. Henthorn PS, Raducha M, Fedde KN, Lafferty MA, Whyte MP. Different missense mutations at the tissue-nonspecific alkaline phosphatase gene locus in autosomal recessively inherited forms of mild and severe hypophosphatasia. Proc Natl Acad Sci U S A. 1992;89:9924-8.

33. Orimo H, Girschick HJ, Goseki-Sone M, Ito M, Oda K, Shimada T. Mutational analysis and functional correlation with phenotype in German patients with childhood-type hypophosphatasia. J Bone Miner Res. 2001;16:2313-9.

34. Ozono K, Yamagata M, Michigami T, Nakajima S, Sakai N, Cai G, et al. Identification of novel missense mutations (Phe310Leu and Gly439Arg) in a neonatal case of hypophosphatasia. J Clin Endocrinol Metab. 1996;81:4458-61.

\section{Publisher's Note}

Springer Nature remains neutral with regard to jurisdictional claims in published maps and institutional affiliations.

Ready to submit your research? Choose BMC and benefit from:

- fast, convenient online submission

- thorough peer review by experienced researchers in your field

- rapid publication on acceptance

- support for research data, including large and complex data types

- gold Open Access which fosters wider collaboration and increased citations

- maximum visibility for your research: over $100 \mathrm{M}$ website views per year

At $\mathrm{BMC}$, research is always in progress.

Learn more biomedcentral.com/submissions 